\title{
Immunosuppression by pulmonary surfactant: mechanisms of action
}

\author{
Margaret L Wilsher, David J Parker, Patricia L Haslam
}

\begin{abstract}
Pulmonary surfactant has been shown by this group to suppress peripheral blood lymphocyte responses to mitogens and alloantigens in a dose dependent manner, though the mechanism of action of the suppressive effect is not clearly understood. To try to clarify this, attempts were made to reverse the effects of preincubation with surfactant, obtained by bronchoalveolar lavage from pigs, on lymphocytes and accessory monocytes obtained from the blood of normal volunteers, by washing and incubating the cells in medium alone for various periods up to 24 hours. Immunosuppression, measured as the reduction in thymidine incorporation in response to the mitogen phytohaemagglutinin, could not be reversed by these methods. The addition of indomethacin (up to $100 \mu \mathrm{g} / \mathrm{ml}$ for 72 hours) also had no effect, indicating that the immunosuppression was not related to synthesis of prostaglandins. Incubation with surfactant for as little as two hours before addition of mitogen suppressed in vitro lymphoproliferative responses by half, but surfactant added two hours after mitogen had no observed effect. Preincubation of purified lymphocytes in surfactant, before they were cultured with accessory monocytes and mitogen, caused significant suppression of response, but preincubation of purified monocytes had no suppressive effect. There was no change in the intensity of HLA-DR expression on monocytes. These results support the hypothesis that surfactant exerts its effects on the resting uncommitted lymphocyte rather than on antigen presenting monocytes.
\end{abstract}

An immunoregulatory role for surfactant would be potentially beneficial in vivo in protecting the delicate gas exchanging regions of the lung from unwanted immune reactions to inhaled particles and microorganisms. We have previously reported an immunosuppressive function for normal pulmonary surfactant from man, pig, and rabbit in vitro. ${ }^{1}$ Similar in vitro suppressive effects have been reported for canine pulmonary surface active material and for serum lipoproteins. ${ }^{2-4}$ These effects, which are dose dependent and species independent, are observed for a range of specific primary immune responses: lymphoproliferation in response to mitogens and alloantigens, B cell immunoglobulin production, and natural killer cell cytotoxic function. ${ }^{56}$ Whereas the major surfactant components, phosphatidyl choline, phosphatidylglycerol and phosphatidylinositol exert similar suppressive effects on lymphoproliferation, some of the lesser componentsphosphatidylethanolamine, sphingomyelin, and cholesterol-have been shown to augment lymphoproliferative responses under certain conditions. ${ }^{7}$ Thus the role of surfactant in regulating local immune responses in vivo may be determined by the relative proportions of "suppressive" and "stimulatory" lipid components, and these may be altered in various pulmonary diseases. ${ }^{8}$

Despite the potential importance of these effects, several important questions remain unanswered. Firstly, the mechanism of surfactant induced immune suppression has not been adequately examined, and we do not know whether the effect can be reversed. Secondly, it is not clear whether surfactant exerts its suppressive effect on the antigen presenting cell or the responding lymphocyte. This information is needed before we can consider whether therapeutic manipulation of the surfactant system might benefit patients with inflammatory lung diseases.

Alveolar macrophages function relatively poorly as accessory cells in vitro. ${ }^{910}$ The importance of local influences such as pulmonary surfactant in determining why alveolar macrophages behave in this way has received little attention. Curtiss et $a l^{11}$ have shown that low density lipoprotein in serum exerts its suppressive effects on the responder lymphocyte rather than the stimulator lymphocyte or accessory cell macrophage in mixed lymphocyte cultures. Whether pulmonary surfactant can exert an effect on the function of accessory cells as well as responder cells, and whether this might partly explain the poor accessory cell function of alveolar macrophages, is not known. We sought to examine this in a manner similar to Curtiss $e t$ al $l^{11}$ using a mitogen driven system, and we also looked at monocyte MHC class II region product expression after incubation with surfactant because of the important role of these cell surface molecules in $\mathrm{T}$ cell recognition of antigens.

The aim of this study therefore was, firstly, to examine in greater detail the immunosuppressant effects of pulmonary surfactant and in particular to explore at what stage of cellular activation surfactant exerts its effects; secondly, to determine whether the effects can be reversed by washing or by indomethacin; 
and, thirdly, to explore whether the lymphocyte or the accessory monocyte is the target cell for suppression.

\section{Methods}

PURIFICATION OF SURFACTANT

Bronchoalveolar lavage fluid from three pigs was subjected to methanol chloroform extraction and reconstituted by ultrasonication into medium as previously described. ${ }^{1}$ This preparation, which contains the normal lipid components in proportions generally accepted for pulmonary surfactant, ${ }^{12}$ has been shown to have surface active properties. ${ }^{13}$ The phospholipid profiles of the surfactant from each pig were determined with a one dimensional thin layer chromatography system to separate the individual phospholipids, followed by charring and quantitation by reflectance densitometry ${ }^{1}$ (table). Phosphatidylcholine and cholesterol were obtained from a commercial source (Sigma) and also reconstituted as previously described. $^{7}$

\section{PREPARATION OF PERIPHERAL BLOOD} LYMPHOCYTES

Whole blood was obtained from healthy adult volunteers and separated on Ficoll-Hypaque as previously described ${ }^{1}$ and the peripheral blood mononuclear cells were resuspended in serum free medium (RPMI) containing the following additives: selenium $(6.25 \mathrm{ng} / \mathrm{ml})$, transferrin $(6.25 \mu \mathrm{g} / \mathrm{ml})$, insulin $(6.25 \mu \mathrm{g} / \mathrm{ml})$, linoleic acid $(5.35 \mu \mathrm{g} / \mathrm{ml})$, bovine serum albumin $(1.25 \mathrm{mg} /$ $\mathrm{ml})$. For use in the accessory cell experiments blood mononuclear cells were further separated into pure lymphocyte (Ly) and pure monocyte (M) populations as follows. After adherence for one hour in serum free medium in polystyrene flasks to allow attachment of monocytes, the non-adherent cells (mainly lymphocytes) were aspirated, washed twice and incubated with sheep red blood cells (treated with 2-aminoethylisothiouronium) for 20 minutes at $37^{\circ} \mathrm{C}$ to allow rosette formation with the $\mathrm{T}$ lymphocytes. The cells were then centrifuged at $300 \mathrm{~g}$ for 10 minutes and placed on ice for one hour. The pellet was then gently resuspended and layered over Ficoll-Hypaque before being centrifuged at $500 \mathrm{~g}$ for 20 minutes. The upper layers were discarded and the pellet containing rosetted $\mathrm{T}$ lymphocytes was resuspended in $10 \mathrm{mM}$ ammonium chloride for five minutes to lyse the sheep red blood cells. After red cell lysis the T lympho-

Phospholipid profiles of surfactant from three pigs used in the experiments

\begin{tabular}{lrrrr}
\hline & \multicolumn{3}{c}{ Pig No } & \multicolumn{1}{c}{$\begin{array}{c}\text { Mean } \\
\text { (SD) }\end{array}$} \\
\cline { 2 - 4 } Phospholipid $\left({ }^{\circ}{ }_{0}\right)$ & 1 & 2 & 3 & \\
\hline Lysophosphatidylcholine & 0 & 12 & 8 & $7(6)$ \\
Sphingomyelin & 22 & 9 & 11 & $14(7)$ \\
Phosphatidylcholine & 50 & 62 & 60 & $57(6)$ \\
Phosphatidylinositol & 11 & 0 & 5 & $5(5)$ \\
Phosphatidylserine & 0 & 0 & 0 & 0 \\
Phosphatidylethanolamine & 4 & 2 & 3 & $3(1)$ \\
Phosphatidylglycerol & 13 & 15 & 13 & $14(1)$ \\
Cardiolipin & 0 & 0 & 0 & 0 \\
\hline
\end{tabular}

cytes were washed three times, resuspended in serum free medium, and incubated in a nylon wool column for 45 minutes at $37^{\circ} \mathrm{C}$ to remove any residual contaminating adherent cells. The column was then flushed three times with warmed medium and the recovered lymphocytes were counted and resuspended in medium. The adherent layer of monocytes in the polystyrene flasks was washed four times and the cells were gently removed with a rubber policeman and resuspended in medium. The purity of both populations was tested by non-specific esterase staining and monoclonal antibody labelling (Leu 4, pan-T cell; Leu 12, pan-B cell; and HLA-DR from Becton Dickinson) with subsequent analysis using a fluorescent activated cell sorter (FACS IV Becton Dickinson). The purity of the monocyte population on the basis of non-specific esterase staining was over $87 \%$; it also contained $<8 \%$ Leu 4 and $>70 \%$ HLA-DR positive cells. The lymphocyte population contained $>99.9 \%$ lymphocytes on the basis of non-specific esterase and $>82 \%$ Leu $4,<1 \%$ Leu 12 and $<5 \%$ HLA-DR positive cells. Cell viability was assessed by trypan blue exclusion.

\section{EXPERIMENTAL PROCEDURES}

\section{Preincubation cultures}

Peripheral blood mononuclear cells $\left(0.5 \times 10^{6}\right.$ cells $/ \mathrm{ml}$ ) were preincubated with phytohaemagglutinin $(0.5 \mu \mathrm{g} / \mathrm{ml})$ before the addition of surfactant lipids $(0.1 \mathrm{mg} / \mathrm{ml})$ or with surfactant and then washed twice before the addition of phytohaemagglutinin. The cells were preincubated for varying lengths of time with either substance $(2,4,8,12$ hours). Control cultures contained phytohaemagglutinin alone or phytohaemagglutinin and lipids for the duration of the culture. The concentration of phytohaemagglutinin used in these experiments $(0.5 \mu \mathrm{g} / \mathrm{ml})$ had previously been found to cause optimal lymphoproliferation. All cultures were conducted in triplicate in $6 \mathrm{ml}$ polypropylene tubes (Falcon 2063). Each culture contained $0.5 \times 10^{6}$ cells in $1 \mathrm{ml}$ of serum free medium plus antibiotics (penicillin $100 \mathrm{IU} / \mathrm{ml}$, streptomycin $100 \mu \mathrm{g} / \mathrm{ml}$ ) and were labelled with tritiated thymidine $(37 \mathrm{KBq}) 18$ hours before harvesting. Seventy two hours after the addition of phytohaemagglutinin the cell suspensions were divided into aliquots of $200 \mu \mathrm{l}$ in 96 well microtitre plates and harvested with an Ilacon harvester. The results of counts from triplicate cultures varied by less than $15^{\circ} \%$ from the mean value for each subject.

Reversibility of surfactant induced suppression Peripheral blood mononuclear cells $\left(0.5 \times 10^{6}\right.$ cells $/ \mathrm{ml}$ ) were incubated in serum free medium containing surfactant lipids $(0.1 \mathrm{mg} / \mathrm{ml})$ for four hours. The cells were then washed twice and reincubated in serum free medium alone for varying lengths of time $(4,12,24$ hours) before the addition of phytohaemagglutinin. For comparison cultures were also performed without the washing step, the lipids remaining for the duration of culture after the addition of phytohaemagglutinin after four hours' preincubation. Peripheral blood mononuclear 
cells were also preincubated in either phosphatidylcholine or cholesterol for four hours, washed twice, then incubated in the other lipid for four hours and washed twice before the addition of phytohaemagglutinin. The cells were harvested 72 hours after the addition of phytohaemagglutinin to the cultures as previously described. Control cultures with or without surfactant lipids were incubated with phytohaemagglutinin for 72 hours without any preincubation or washing steps. Cells were recounted after the two washing steps and greater than $90 \%$ recovery was always observed.

\section{Effect of indomethacin}

Peripheral blood mononuclear cells $\left(0.5 \times 10^{6}\right.$ cells $/ \mathrm{ml}$ ) from three subjects were cultured in either phosphatidylcholine $(0.2 \mathrm{mg} / \mathrm{ml})$ or surfactant lipids $(0.2 \mathrm{mg} / \mathrm{ml})$ together with optimal phytohaemagglutinin $(0.5 \mu \mathrm{g} / \mathrm{ml})$ and increasing concentrations of indomethacin ( 1 , $10,100 \mu \mathrm{g} / \mathrm{ml}$, Sigma). Cultures were performed in both serum-containing and serum free medium and harvested at 72 hours as above.

Surfactant effects on monocytes and lymphocytes Pure lymphocytes (Ly) and pure monocytes (M) were cultured separately overnight in the presence $(\mathrm{Ly}+, \mathrm{M}+)$ or absence $(\mathrm{Ly}-, \mathrm{M}-$ ) of surfactant lipids $(0 \cdot 1 \mathrm{mg} / \mathrm{ml})$. After washing three times, monocytes preincubated with $(\mathrm{M}+)$ or without $(\mathbf{M}-)$ surfactant were added to lymphocytes preincubated with $(\mathrm{Ly}+)$ or without $(\mathrm{Ly}-)$ surfactant in a ratio of $1: 5$, making a total of $0.6 \times 10^{6} \mathrm{cells} / \mathrm{ml}$ in serum free medium. Four sets of cultures were performed: $\mathrm{Ly}+\mathrm{M}+, \mathrm{Ly}+\mathrm{M}-, \mathrm{Ly}-\mathrm{M}+$, Ly $-\mathbf{M}-$. Lymphocytes were also cultured alone to determine the accessory cell dependence of the system. All cultures were performed in triplicate in the presence of optimal phytohaemagglutinin and continued for 72 hours as above. Results were expressed as percentages of the proliferation in the control cultures without surfactant $(\mathrm{Ly}-\mathrm{M}-)$.

\section{STATISTICAL ANALYSIS}

Results for pairs of related samples were compared by means of the Wilcoxon matched pairs signed ranks test. Values of $p$ of 0.05 or less (two tailed) were accepted as significant.

\section{Results}

\section{PREINCUBATION CULTURES}

Spontaneous uptake of tritiated thymidine in control cultures of peripheral blood mononuclear cells without phytohaemagglutinin was not significantly different in the presence and in the absence of added surfactant lipids. Culture of peripheral blood mononuclear cells in surfactant lipids for as little as two hours before the addition of mitogen resulted in substantial suppression of the proliferative response to phytohaemagglutin-to less than half of the proliferation obtained in control lipid free cultures not subjected to the preincubation steps (fig 1). This was equivalent to the level of.

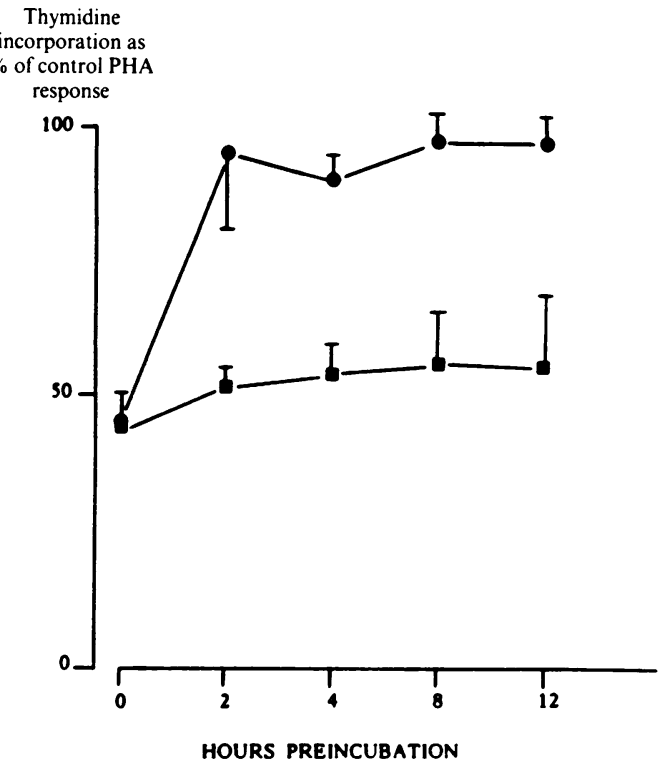

Figure 1 Effect on the lymphoproliferative response to the mitogen phytohaemagglutinin (PHA, $0.5 \mu \mathrm{g} / \mathrm{ml})$ of preincubating peripheral blood mononuclear cells from four subjects for 4, 12, or 24 hours either with surfactant lipids before the addition of phytohaemagglutinin ( $\mathbf{0}$ ) or with phytohaemagglutinin before the addition of surfactant lipids (O). Results were calculated for each subject as percentages of the phytohaemagglutinin induced proliferation obtained in lipid free control cultures for the same subject, which were not subjected to preincubation steps. The figure shows the mean (SEM) percentage of the control response for the group at each time point. (The mean (SEM) thymidine incorporation for the lipid free control cultures was 8367 (1838) cpm with phytohaemagglutinin and 912 (389) cpm without phytohaemagglutinin.)

suppression seen if surfactant was added with phytohaemagglutinin at 0 hours and continued throughout culture. If, however, surfactant was added two hours or more after the addition of phytohaemagglutinin to the cultures no suppression was observed (fig 1). Cell viability was not affected by addition of surfactant to cultures.

\section{REVERSIBILITY OF SURFACTANT AND LIPID EFFECTS}

The suppressive effects of surfactant were not reversed significantly by washing and incubation in medium alone for 4,12 , or 24 hours (points 3, 4, and 5 on the horizontal axis of fig 2) when compared with the lipid containing cultures set up without washing (first and second points on the horizontal axis; Wilcoxon matched pairs signed ranks test). There was a tendency, though it was not significant, towards increasing responses to phytohaemagglutinin after 12 and 24 hours (fig 2). Incubation of cells with phosphatidylcholine for four hours resulted in suppression of the phytohaemagglutinin response that was not reversed by reincubation in cholesterol. Conversely, the stimulatory effect of cholesterol ${ }^{4}$ was only variably observed after a four hours' incubation; any possible effect of phosphatidylcholine in reversing the stimulatory effect of cholesterol could not be determined in these experiments.

\section{EFFECT OF INDOMETHACIN}

Addition of indomethacin to serum free cul- 


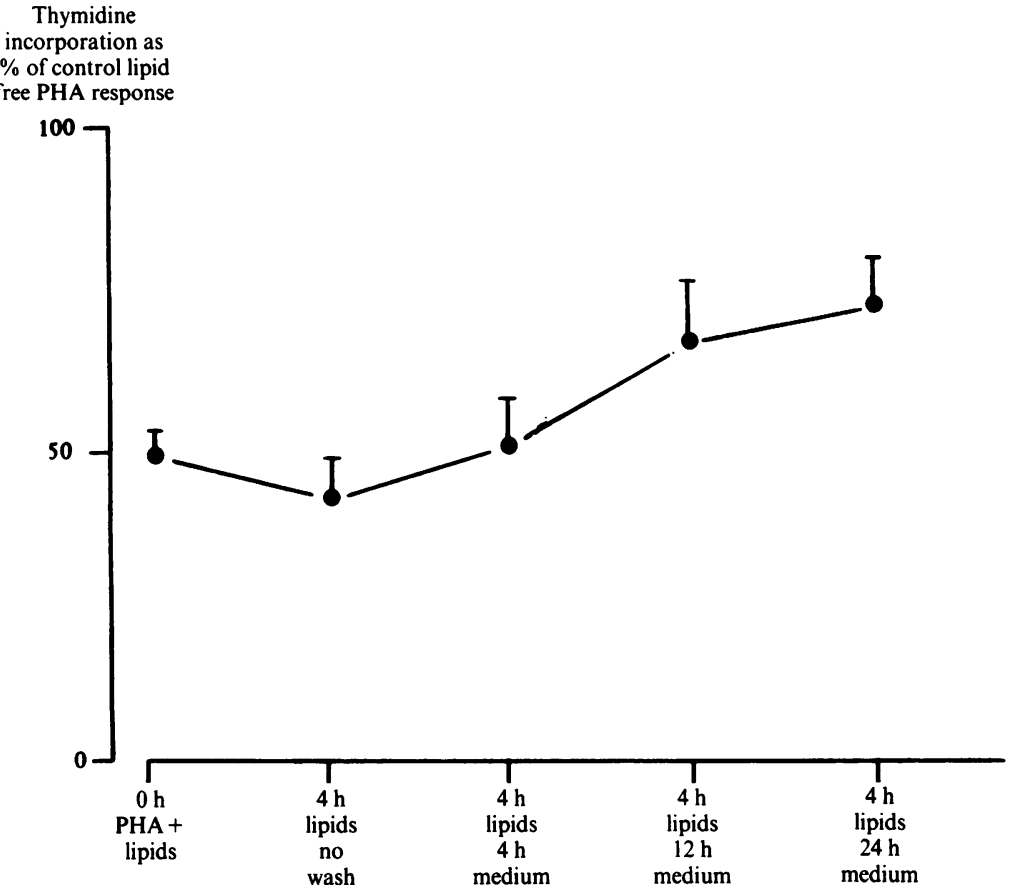

BEFORE THE ADDITION OF PHA

Figure 2 Graph showing that washing (points 3, 4 and 5 on the horizontal axis) did not significantly reverse the suppressive effect of surfactant lipids (four hours prior incubation) on the proliferative response of peripheral blood mononuclear cells to the mitogen phytohaemagglutinin (PHA, 0.5 $\mu \mathrm{g} / \mathrm{ml}$ ) (point 2 on the horizontal axis) (4 subjects, Wilcoxon matched pairs signed ranks test). Results were calculated for each subject as percentages of the proliferation achieved with control peripheral blood mononuclear cells for the same subject not exposed to lipids or subjected to washing and incubation steps. The figure shows the mean (SEM) percentage control response for the group at each point studied. (The mean (SEM) thymidine incorporation for the lipid free control cultures was 19694 (4726) cpm with phytohaemagglutinin and 1008 (360) cpm without phytohaemagglutinin.)

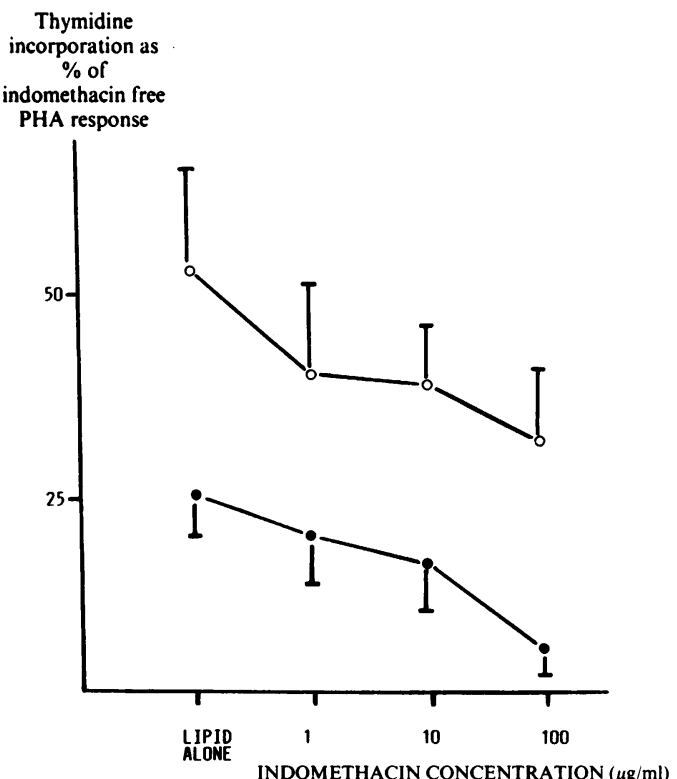

Figure 3 Effect of indomethacin on the suppressive effects of surfactant lipids, $0.2 \mathrm{mg} / \mathrm{ml}(\mathbf{O})$, or phosphatidylcholine, $2 \mathrm{mg} / \mathrm{ml}(\mathrm{O})$, on the proliferative response of peripheral blood lymphocytes to the mitogen phytohaemagglutinin $(P H A, 0.5 \mu g / m l)(n=3)$. The results were calculated for each subject as percentages of the phytohaemagglutinin induced proliferation achieved in control cultures for the same subject not exposed to lipids or indomethacin. The figure shows the mean (SEM) percentage control response for the group at each point. (The mean (SEM) thymidine incorporation for the lipid and indomethacin free phytohaemagglutinin control cultures was 32193 (9339) in serum free medium and 21584 (4752) in serum containing medium.) tures containing surfactant $(0.2 \mathrm{mg} / \mathrm{ml})$ did not reverse the suppressive effect of surfactant but caused additional suppression with increasing amounts of added indomethacin (fig 3). Similarly, the addition of indomethacin to cultures containing phosphatidylcholine $(2 \mathrm{mg} / \mathrm{ml})$ in serum containing medium also caused more suppression than occurred in the cultures containing phosphatidylcholine alone (fig 3). Indomethacin itself was also found to cause some suppression of the lymphocyte response to phytohaemagglutinin in serum free cultures without added lipid (in the presence of 1,10 , and $100 \mu \mathrm{g} / \mathrm{ml}$ indomethacin, the response to phytohaemagglutinin was $70 \%$, $68 \%$, and $14 \%$ of the response of control, indomethacin free cultures; data not shown in fig 3). Cell viability was not affected by the indomethacin or lipids.

SURFACTANT EFFECTS ON PURE MONOCYTES AND PURE LYMPHOCYTES

The phytohaemagglutinin response of cultures in which monocytes but not lymphocytes were preincubated in surfactant lipids $(\mathbf{L y}-\mathbf{M}+$ ) was no less than the response in control cultures in which neither lymphocytes nor monocytes had encountered surfactant ( $\mathrm{Ly}-$ $M-$ ) (fig 4). The response of cultures in which lymphocytes but not monocytes had been preincubated with surfactant (Ly $+\mathbf{M}-)$, however, was significantly less than that of control cultures $(p=0.01$, Wilcoxon matched pairs signed ranks test). The degree of

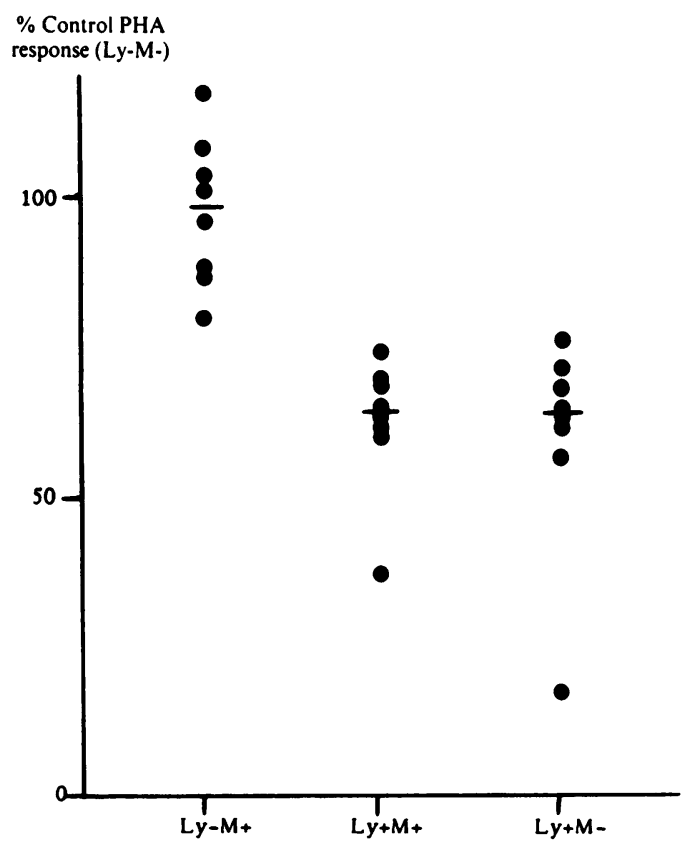

Figure 4 Effect of separately preincubating purified lymphocytes $(L y)$ and $/$ or monocytes $(M)$ (accessory cells) with surfactant $(L y+, M+)$ or without surfactant (Ly-,M-) before mixing them to test their

proliferative response to phytohaemagglutinin (PHA) in eight subjects. Results are expressed as percentages of the phytohaemagglutinin induced proliferation obtained in control cultures without surfactant contact $(L y-M-)$ for each subject. The reduction in response in the $L y+$ $M+$ and $L y+M-$ groups compared with controls was significant ( $p=0.01$, Wilcoxon matched pairs signed rank test). (The mean (SEM) thymidine incorporation for the lipid free control cultures was $11753(2646) \mathrm{cpm}$ with phytohaemagglutinin and 605 (178) cpm without phytohaemagglutinin.) 
suppression was similar to that in cultures where both lymphocytes and monocytes had been preincubated with surfactant $(\mathrm{Ly}+\mathrm{M}+)$ (mean \% suppression 60 (SEM 6) compared with 63 (4)), suggesting that lymphocytes but not monocytes were affected by preincubation with surfactant (fig 4). Cultures of lymphocytes in the absence of monocytes responded only minimally to phytohaemagglutinin, confirming the accessory cell dependence of the system.

There was no change in the percentage HLA-DR expression of the monocyte population after culture in surfactant (mean 86 (SD 5) compared with 87 (5)).

\section{Discussion}

These results suggest that pulmonary surfactant exerts a largely irreversible suppressive influence at an early stage in the initiation of lymphoproliferation in vitro as both washing and reincubating in fresh medium failed to overcome the effects of surfactant. Once a cell is committed to entry into the cell cycle and proliferation, however, surfactant has no suppressive effect, as shown by those experiments in which surfactant was added after contact of the cells with mitogen. Moreover, the suppressive effect seems wholly directed at the lymphocyte, because the accessory cell (monocyte) in these experiments was not affected by incubation with surfactant, nor was there any change in expression of MHC class II (HLADR) antigens. These experiments do not therefore support a role for surfactant in depressing the observed in vitro accessory cell function of alveolar macrophages.

We acknowledge that the mitogen driven system used in these experiments does not specifically examine antigen processing and presentation. Accessory cells, however, are required for lymphocyte responses to mitogen and they cannot be replaced by adding exogenous interleukin 1 to culture. ${ }^{14}{ }^{15}$ Intact accessory cells may be necessary to provide a matrix facilitating the cross linking of $T$ cell surface receptors by phytohaemagglutinin as cell to cell contact is required for interleukin 2 responsiveness. ${ }^{16}$ We went to great lengths to exclude accessory cells from the pure lymphocyte population and have shown that the system is dependent on accessory cells. A mitogen driven system was used for two reasons: firstly, the lymphocyte responses to mitogen are greater and more consistent than with antigen and, secondly, it was essential that the cultures were performed in serum free culture medium to avoid any effects from serum lipids. Such medium will support lymphocyte responses to a far smaller extent than medium containing serum and optimally for generally less than four or five days. Thus the overall responses to antigen under such conditions are likely to be variable.

Indomethacin was added to cultures in an attempt to determine whether surfactant might be stimulating the production of metabolites of the arachidonic acid pathway, such as prostaglandin $\mathrm{E}_{2}$, by monocytes, and whether these products might be mediating the suppressive effects of superfactant on the lymphocyte. Indomethacin treatment failed to reverse the suppressive effects so our results do not suggest that surfactant enhances prostaglandin $E_{2}$ production. Our findings confirm that indomethacin itself causes suppression of mitogenesis at the concentrations we used, whereas variable enhancement has been reported at lower concentrations $(<3 \mu \mathrm{mol} / \mathrm{l}){ }^{17}{ }^{18}$ The poor accessory cell function of alveolar macrophages has been attributed in part to prostaglandin $\mathrm{E}_{2}$ production, which then suppresses lymphocyte responses. ${ }^{19}$ Our results, however, do not support any effect of surfactant either on monocyte accessory cell function or on prostaglandin $\mathrm{E}_{2}$ production, strengthening the theory that the lymphocyte is the cell directly influenced by surfactant in vitro.

An early irreversible suppressive effect on the lymphocyte is consistent with the idea that surfactant exerts its influences via changes in the cell membrane, with subsequent effects on signal transmission and entry into the cell cycle. It is generally acknowledged that an optimal cell membrane fluidity is necessary for optimal cellular function and homeostatic regulation of the membrane lipid composition is rigorously controlled. ${ }^{20}$ Membrane fluidity in certain cells is altered by changing the ratios of saturated to unsaturated fatty acids, sphingomyelin to phosphatidylcholine (lecithin), and cholesterol to phospholipids in the culture medium. ${ }^{2122}$ Incubation of lymphocytes with cholesterol and phosphatidylcholine liposomes alters not only membrane fluidity but also responsiveness to mitogen. ${ }^{2324}$ Generally speaking, exogenous cholesterol will decrease membrane fluidity, with an increase in the responsiveness of lymphocytes to phytohaemagglutinin, whereas phosphatidylcholine will have the opposite effect. In view of these findings, ${ }^{7}$ we formed the hypothesis that the membrane effects of phosphatidylcholine could be reversed by subsequent culture in cholesterol and those of cholesterol by culture in phosphatidylcholine, with the effects reflected in enhanced and decreased responsiveness to mitogen. The experiments in this study do not support this, however, and suggest that exogenous lipids in culture have a largely irreversible effect on cell membrane dynamics. It remains to be determined whether the effect of surfactant lipids on the $\mathrm{T}$ lymphocyte interferes with antigen recognition, binding to accessory cells, or signal transmission.

The observed effects of surfactant in suppressing in vitro immune responses may account for the lack of observed specific immune activity in the alveolus despite the constant challenges of inhaled antigens. Nonspecific innate immune functions, such as phagocytosis and lysis of microorganisms, remain intact, however, and surfactant has been reported as enhancing these functions in vitro. ${ }^{25}$ These potentially beneficial effects could be put to therapeutic use in certain pulmonary conditions in which there is a quantitative or qualitative deficiency of surfactant, such as the neonatal and adult respiratory 
distress syndrome. Some understanding of the possible mechanism of action of surfactant in suppressing immune responses is therefore important, especially as artificial surfactants are gaining popularity in the treatment of such disorders. ${ }^{26}$

In summary, having previously shown that pulmonary surfactant has immunosuppressive properties in vitro, we have shown in this study that the effects are directed at the lymphocyte at the stage of cell activation rather than proliferation. We postulate that a change in the cell membrane may account for this.

We wish to thank Mr David Hughes and $\mathrm{Mr}$ Philip Townsend for scientific assistance and Mrs Joanna Harwood for typing the manuscript. This work was supported by The Wellcome Trust and The Medical Research Council of New Zealand. Our work in flow cytometry was supported by the British Lung Foundation and the Percy Bilton Charity.

1 Wilsher ML, Hughes DA, Haslam PL. Immunoregulatory properties of pulmonary surfactant: effect of lung lining properties of pulmonary surfactant: effect of lung lining
fluid on proliferation of human blood lymphocytes. Thorax 1988;43:354-9.

2 Morse JH, Witte LD, Goodman De WS. Inhibition of lymphocyte proliferation stimulated by lectins and allogenic cells by normal plasma lipoproteins. J Exp Med 1977;146:1791-803.

3 Curtiss LK, Edgington TS. Regulatory serum lipoproteins: regulation of lymphocyte stimulation by a species of low density lipoprotein. J Immunol 1977;199:2129-36.

4 Ansfield MJ, Kaltreider HB, Benson BJ, Coldwell JL. Immunosuppressive activity of canine surface active materials. J Immunol 1979;122:1062-6.

5 Wilsher ML, Hughes DA, Haslam PL. Immunomodulatory effects of pulmonary surfactant on natural killer cell and antibody dependent cytotoxicity. Clin Exp Immunol 1988;94:465-70.

6 Sitrin RG, Ansfield MJ, Kaltreider HB. The effect of pulmonary surface active material on the generation of murine $\mathrm{B}$ and $\mathrm{T}$ lymphocyte functions in vitro. Exp Lung Res 1985;9:85-97.

7 Wilsher ML, Hughes DA, Haslam PL. Immunoregulatory properties of pulmonary surfactant. (ii) Influence of variations in the phospholipid profile. Clin Exp Immunol variations in the

8 Hughes DA, Haslam PL. Changes in phosphatidylglycerol in bronchoalveolar lavage fluids from patients with cryptogenic fibrosing alveolitis. Chest 1989;95:82-9.
9 Ettensohn DB, Roberts NJ. Human alveolar macrophage support of lymphocyte responses to mitogens and antigens. Am Rev Respir Dis 1983;128:516-22.

10 Lipscomb MF, Lyons CR, Nunex G, et al. Human alveolar macrophages: HLA-DR positive macrophages that are poor stimulators of a primary mixed leucocyte reaction. $J$ Immunol 1986;136:497-504.

11 Curtiss LK, Edgington TS. Effect of LDL-In, a normal immunoregulatory human serum low density lipoprotein on the intereaction of macrophages with lymphocyte proliferating in response to mitogen and allogeneic stimulation. J Immunol 1977;118:1966-70.

12 King RJ. Pulmonary surfactant. J A ppl Physiol 1982;53:1-8.

2 Kin RJ. Pulmonary surfactant. J Appl Physiol 1982;53:1-8. Jenkin G. Physical and physiological properties of dry Jenkin G. Physical and physiological p
lung surfactant. Nature 1979;271:162-3.

14 Grey HM, Chestnut R. Antigen processing and presentation to T-cells. Immunology Today 1985;6:101-6.

15 Davis L, Lipsky PE. Signals involved in T-cell activation. II. Distinct roles of intact accessory cells, phorbol esters and interleukin 1 in activation and cell cycle progression of resting T-lymphocytes. J Immunol 1986;136:3588-96.

16 Hunig $\mathrm{T}$. The role of accessory cells in polyclonal T-cell activation. II. Induction of interleukin 2 responsiveness requires cell-cell contact. Eur J Immunol 1983;13 596-601.

17 Demenkoff JH, Ansfield MJ, Kaltreider HB, Adam E. Alveolar macrophage suppression of canine bronchoalveolar lymphocytes: the role of prostaglandin $E$ in the inhibition of mitogen responses. J Immunol 1980;124:1365-70.

18 Kelly JP, Johnson MC, Parker CW. Effect of inhibitors of arachidonic acid metabolism on mitogenesis in human lymphocytes: possible role of thromboxanes and products of the lipoxygenase pathway. J Immunol 1979;122: 1563-71.

19 Rich EA, Tweardy DJ, Fujiwara H, Ellner JJ. Spectrum of immunoregulatory functions and properties of human alveolar macrophages. Am Rev Respir Dis 1987;136: 258-65.

20 Singer SJ, Nicholson GL. The fluid mosaic model of the structure of cell membranes. Science 1972;175:720-31.

21 Shinitzky M, Inbar M. Difference in microviscosity induced by different cholesterol levels in the surface membrane layer of normal lymphocytes and malignant lymphoma cells. J Mol Biol 1974;85:603-15.

22 Stubbs CD, Smith AD. The modification of mammalian membrane polyunsaturated fatty acid composition in relation to membrane fluidity and function. Biochim Biophys Acta 1984;779:89-137.

23 Rivnay B, Globerson A, Shinitzky M. Perturbation of lymphocyte response to concanavalin $\mathrm{A}$ by exogenous cholesterol and lecithin. Eur J Immunol 1978;8:185-9.

24 Shine TE, Little JR, Shore V, Medoff G, Abegg A. Effect of exogenous lipids and lipoproteins on the primary immune response in vitro. Eur J Immunol 1980;10:714-8.

25 O'Neill, Lesperance E, Klass DH. Rat lung lavage surfactant enhances bacterial phagocytosis and intracellular killing by alveolar macrophages. Am Rev Respir Dis 1984; by alveolar

26 Jobe A, Ikegami M. Surfactant for the treatment of respiratory distress syndrome. Am Rev Respir Dis 1987;136:1256-75. 